\title{
Editorial
}

\section{Challenges and Opportunities for Lung Ultrasound in Novel Coronavirus Disease (COVID-19)}

\author{
Marcus J. Schultz, ${ }^{1,2,3 *}$ Chaisith Sivakorn, ${ }^{4}$ and Arjen M. Dondorp ${ }^{1,2,3}$ \\ ${ }^{1}$ Mahidol-Oxford Tropical Medicine Research Unit (MORU), Mahidol University, Bangkok, Thailand; ${ }^{2}$ Nuffield Department of Medicine, Mahidol \\ University, Bangkok, Thailand; ${ }^{3}$ Department of Intensive Care, Amsterdam University Medical Centers, Amsterdam, The Netherlands; ${ }^{4}$ Department \\ of Clinical Tropical Medicine, Faculty of Tropical Medicine, Mahidol University, Bangkok, Thailand
}

Novel coronavirus disease (COVID-19) is responsible for hundreds of thousands of hospitalizations worldwide, and this number is still increasing rapidly in many countries as of midApril 2020. Diagnostic and clinical approaches differ between countries and even within countries because of inconsistent presence of testing materials and variable access to chest imaging. Severe acute respiratory syndrome-coronavirus-2 reverse transcriptase-polymerase chain reaction from nasopharyngeal or throat swabs has a high specificity but moderate sensitivity. Although patients with COVID-19 can present with a normal chest X-ray early in the disease, ${ }^{1}$ abnormalities on chest images have a higher sensitivity to detect the disease than molecular testing, but lack specificity. ${ }^{2}$ Typical findings on a chest $X$-ray include bilateral and multi-lobar infiltrates that can progress rapidly over the first days of illness. Chest X-ray is the most commonly used imaging technique in suspected COVID-19 in resource-limited settings. However, the image quality is often poor, and follow-up chest X-rays are difficult in settings with constraints on infrastructure, human resources, and personal protective equipment.

Chest computed tomography (CT) scan is more sensitive that chest X-ray to detect early COVID-19. Typical findings on chest CT include bilateral and multi-lobar "ground-glass" or "crazy-paving" opacities, surrounded by spared lung tissue. . $^{3,4}$ Ground-glass and crazy-paving opacities can, however, also be seen in many other pulmonary diseases. The COVID-19 working group of the Dutch Radiological Society recently coined the "CO-RADS classification" for diagnosing COVID-19, grading the level of suspicion for COVID-19 from very low ("CO-RADS 1") to very high ("CO-RADS 5"). ${ }^{5}$ Chest CT scan findings, however, are of limited value in resource-limited settings where access to CT scanners is in general very limited. Chest CT scan access may even be limited in resource-rich settings when hospital systems are overwhelmed with huge numbers of patients with suspected COVID-19. In addition, obtaining a chest CT scan is highly impractical once a patient is intubated and ventilated because transportation to the CT scanner is labor intensive and not without risk. Last, but not least, an additional burden is that the CT scanner needs to be thoroughly cleaned after each suspected case of COVID-19, to prevent the spread of the infection to other patients and healthcare personnel.

Point-of-care lung ultrasound (LUS) could play an important role in COVID-19 management. Lung ultrasound is a noninvasive, rapid, repeatable, and sensitive bedside method to detect a range of pulmonary (and extrapulmonary) pathologies, including pneumothorax, pleural effusions, pulmonary infiltrates, and lung consolidations. In this issue of the American

* Address correspondence to Marcus J. Schultz, Amsterdam University Medical Centers, Meibergdreef 9, 1105 AZ Amsterdam, The Netherlands. E-mail: marcus.j.schultz@gmail.com
Journal of Tropical Medicine and Hygiene, Yasukawa and Taro $^{6}$ report their first experience with LUS in a case series of COVID-19 patients in the United States. All patients had "comet tails" (or "glass rockets"), with or without a "white lung" ("Birolleau variant"), and confluent B lines. Irregular pleural lines were also present in all patients, and half of the patients had small subpleural consolidations. In the one patient with a concomitant chest CT scan, peripheral opacification on the CT scan matched with a white lung area on the LUS images.

The good sensitivity of bedside LUS to detect lung lesions in COVID-19 makes this an attractive additional diagnostic tool in patients suspected of COVID-19, often nursed in isolation rooms or on isolation wards. Lung ultrasound has been implemented for this purpose in several COVID-19-designated hospitals in Thailand. These centers are now also gaining experience in performing daily LUS for early detection of disease progression in COVID19 , with results important for decisions on escalation of care. ${ }^{7}$

One challenge with LUS is defining consistent systems for reporting and interpretation of findings. One frequently used approach is semi-quantification of lung aeration across 12 lung regions into a numerical "global score," in which the presence of A lines counts as " 0 ," less or more $B$ lines count as "1" or "2," and consolidation counts as " 3. ." Scores are then summed to a global score that ranges from 0 (i.e., "no abnormalities at all") to 36 (i.e., "consolidations in all regions"). Global LUS scores combined with $\mathrm{SpO}_{2} / \mathrm{FiO}_{2}$ in a resourcelimited setting, ${ }^{9}$ and with $\mathrm{PaO}_{2} / \mathrm{FiO}_{2}$ in a resource-rich setting, ${ }^{10}$ showed good performance for diagnosing acute respiratory distress syndrome (ARDS) and for assessing ARDS severity. ${ }^{11}$ Of note, current global LUS scores do not include pleural thickening and subpleural abnormalities, which seem often present in COVID-19. We should evaluate urgently whether a global LUS score has diagnostic and prognostic value in COVID19, whether scores correlate with disease severity and CT findings, and how pleural thickening and subpleural abnormalities should be incorporated in the scoring system.

Lung ultrasound could potentially also be an important tool for optimizing invasive ventilation in patients with severe COVID-19. ${ }^{12}$ Although patients with COVID-19 receiving invasive ventilation will often have non-recruitable lung lesions early on, recruitable lesions may develop later in the disease course. In patients with lung lesions that can be opened up, recruitment maneuvers and higher positive end-expiratory pressure (PEEP) may be beneficial. ${ }^{13}$ It is currently not well defined how these recruitable lung areas can be best identified. Comparing the same lung lesion on chest CT scan at different PEEP levels (10 and $20 \mathrm{~cm} \mathrm{H}_{2} \mathrm{O}$ ) can show whether a lesion becomes aerated (Schultz, personal experience in Amsterdam). However, this procedure is impractical, especially in resource-limited settings. In patients with ARDS, it has been shown that PEEP-induced lung recruitment can be adequately estimated with bedside LUS. ${ }^{14}$ A study to assess LUS as a tool to detect recruitable lung 
lesions in patients with severe COVID-19 is currently underway in Amsterdam, the Netherlands, and Brussels, Belgium.

In summary, the report from Yasukawa and $\operatorname{Taro}^{6}$ shows that LUS is a promising additional lung imaging tool in COVID19 , in particular in settings with limited resources. This easyto-perform bedside tool could prove to be important in diagnosis, prognostication, and follow-up of patients, and might also enable identification of recruitable lung lesions to guide invasive ventilation.

Received April 19, 2020. Accepted for publication April 20, 2020.

Published online April 24, 2020.

Authors' addresses: Marcus J. Schultz and Arjen M. Dondorp, Mahidol-Oxford Tropical Medicine Research Unit (MORU), Mahidol University, Bangkok, Thailand, Nuffield Department of Medicine, Mahidol University, Bangkok, Thailand, and Department of Intensive Care, Amsterdam University Medical Centers, Amsterdam, The Netherlands, E-mails: marcus.j.schultz@gmail.com and arjen@ tropmedres.ac. Chaisith Sivakorn, Department of Clinical Tropical Medicine, Faculty of Tropical Medicine, Mahidol University, Bangkok, Thailand, E-mail: chaisith.siv@mahidol.edu.

This is an open-access article distributed under the terms of the Creative Commons Attribution (CC-BY) License, which permits unrestricted use, distribution, and reproduction in any medium, provided the original author and source are credited.

\section{REFERENCES}

1. Rubin GD et al., 2020. The role of chest imaging in patient management during the COVID-19 pandemic: a multinational consensus statement from the Fleischner Society. Chest. Available at: https://doi.org/10.1016/j.chest.2020.04.003.

2. Bai HX et al., 2020. Performance of radiologists in differentiating COVID-19 from viral pneumonia on chest CT. Radiology. Available at: https://doi.org/10.1148/radiol.2020200823.

3. Salehi S, Abedi A, Balakrishnan S, Gholamrezanezhad A, 2020. Coronavirus disease 2019 (COVID-19): a systematic review of imaging findings in 919 patients. AJR Am J Roentgenol. Available at: https://doi.org/10.2214/AJR.20.23034.
4. Sivakorn C, Luvira V, Muangnoicharoen S, Piroonamornpun $P$, Ouppapong T, Mungaomklang A, lamsirithaworn S, 2020. Case report: walking pneumonia in novel coronavirus disease (COVID-19): mild symptoms with marked abnormalities on chest imaging. Am J Trop Med Hyg 102: 940-942.

5. COVID working group of the Dutch Radiological Society, 2020. COVID-19 CORADS Classification. Available at: https:// radiologyassistant.nl/chest/covid-19-corads-classification. Accessed April 19, 2020.

6. Yasukawa K, Taro M, 2020. Point-of-care lung ultrasound findings in patients with COVID-19 pneumonia. Am J Trop Med Hyg 102: 1198-1202.

7. Department of Disease Control Thailand, 2020. Guidelines for Health Care and Public Health Workers. Available at: https:// ddc.moph.go.th/viralpneumonia/eng/guidelines.php. Accessed April 19, 2020.

8. Volpicelli G et al.; International Liaison Committee on Lung UItrasound (ILC-LUS) for International Consensus Conference on Lung Ultrasound (ICC-LUS), 2012. International evidencebased recommendations for point-of-care lung ultrasound. Intensive Care Med 38: 577-591.

9. Riviello ED, Kiviri W, Twagirumugabe T, Mueller A, BannerGoodspeed VM, Officer L, Novack V, Mutumwinka M, Talmor DS, Fowler RA, 2016. Hospital Incidence and outcomes of the acute respiratory distress syndrome using the Kigali modification of the Berlin definition. Am J Respir Crit Care Med 193: 52-59.

10. Vercesi $V$ et al., 2018. External confirmation and exploration of the Kigali modification for diagnosing moderate or severe ARDS. Intensive Care Med 44: 523-524.

11. Pisani $L$ et al., 2019. The diagnostic accuracy for ARDS of global versus regional lung ultrasound scores - a post hoc analysis of an observational study in invasively ventilated ICU patients. Intensive Care Med Exp 7: 44.

12. Dondorp AM, Mumamed H, Aryal D, Beane A, Schultz MJ, 2020. Respiratory support in COVID-19 patients, with a focus on resource-limited settings. Am J Trop Med Hyg 102: 1191-1197.

13. Schultz MJ, 2020. High vs low PEEP in nonrecruitable collapsed lung tissue: possible implications for patients with COVID-19. Lancet Resp Med. Available at: https://doi.org/10.1016/S22132600(20)30180-6.

14. Bouhemad B, Brisson H, Le-Guen M, Arbelot C, Lu Q, Rouby JJ, 2011. Bedside ultrasound assessment of positive endexpiratory pressure-induced lung recruitment. Am $J$ Respir Crit Care Med 183: 341-347. 